\title{
Successful management of acute myeloid leukemia transformed from chronic myelomonocytic leukemia in the elderly by a combination regimen of decitabine and cytarabine, aclarubicin and granulocyte colony-stimulating factor: A case report
}

\author{
QI DENG, JING-YI LI, PENG-JIANG LIU and MING-FENG ZHAO \\ Department of Hematology, The First Central Hospital of Tianjin, Tianjin 300192, P.R. China
}

Received April 4, 2014; Accepted December 18, 2014

DOI: $10.3892 / \mathrm{ol} .2015 .2870$

\begin{abstract}
Despite advances in the treatment of acute myeloid leukemia (AML) in recent years, the outcome of elderly AML patients with antecedent hematological disorders remains unsatisfactory. The present study describes a case of complete remission in an elderly patient with AML transformed from chronic myelomonocytic leukemia (CMML) and the treatment of the case with decitabine in combination with cytarabine, aclarubicin and granulocyte colony-stimulating factor (CAG). A 70-year-old male was admitted with fever, pruritus and weakness that had been apparent for two weeks, and a two-year history of monocytosis (22.5-27.0\%). Further examinations revealed a hemoglobin level of $106 \mathrm{~g} / 1$, a white blood cell count of $39.52 \times 10^{9} / 1$, a platelet count of $81 \times 10^{9} / 1$, Y chromosome loss and uniparental disomy on chromosomes $4 \mathrm{q}, 2 \mathrm{q}$ and $19 \mathrm{p}$. The patient was diagnosed with AML transformed from CMML, with cytogenetic anomalies. A combination regimen of decitabine and CAG was administered. Subsequent to one cycle, the patient achieved complete remission. The patient was then followed up with three courses of the same regimen and achieved clinical remission, with no evidence of AML relapse. The present study suggests that a combination of low-dose decitabine and CAG may offer a novel and potentially effective treatment regimen for elderly AML patients.
\end{abstract}

\section{Introduction}

Acute myeloid leukemia (AML) refers to a highly heterogeneous group of hematopoietic diseases, which are characterized by clonal accumulation and the growth of immature myeloid

Correspondence to: Dr Qi Deng, Department of Hematology, The First Central Hospital of Tianjin, 24 Fukang Road, Tianjin 300192, P.R. China

E-mail: kachydeng@hotmail.com

Key words: acute myeloid leukemia, chronic myeloid leukemia, decitabine, cytarabine, aclarubicin and granulocyte colony-stimulating factor, single nucleotide polymorphism cells within the bone marrow. At present, the primary treatment for AML is remission induction therapy with multiple, combined chemotherapeutic agents, which aims to reduce the total body leukemic cell population to an undetectable level. This is usually followed by either consolidation chemotherapy or allogeneic hematopoietic stem cell transplantation (HSCT), depending on how well the patient responds to chemotherapy alone and their response to intensive treatments (1). The benefits of this treatment approach vary considerably with patient age and AML subtype, which are two of the most important prognostic factors. Elderly AML patients, particularly those $>65$ years old and with a history of myelodysplastic syndromes (MDS) or antecedent hematological disorders, exhibit unsatisfactory tolerance profiles and disappointing complete remission $(\mathrm{CR})$ and overall survival rates following this conventional approach (2-4). As AML predominantly occurs in older individuals and the global population is rapidly aging, novel treatment strategies are urgently required for elderly patients with AML (5). The present study describes a case of CR without relapse nine months after the administration of a combination regimen of decitabine and cytarabine, aclarubicin and granulocyte colony-stimulating factor (G-CSF) (CAG) in an elderly patient with AML transformed from chronic myelomonocytic leukemia (CMML) (6).

\section{Case report}

In March 2013, a 70-year-old male presented to The First Central Hospital of Tianjin (Tianjin, China) with fever, pruritus and weakness that had been apparent for two weeks, and a two-year history of monocytosis (22.5-27.0\%). The inpatient laboratory tests revealed a hemoglobin $(\mathrm{Hb})$ level of $120 \mathrm{~g} / \mathrm{l}$, a white blood cell (WBC) count of $9.23 \times 10^{9} / 1$ and a platelet count of $122 \times 10^{9} / 1$, but an abnormal monocyte percentage of up to $35.5 \%$ (normal range, $5-10 \%$ ). The anti-inflammatory drug, piperacillin-tazobactam (4.5 g, once every $12 \mathrm{~h}$ ), was administered for three days, however, no improvement was noted with regard to the symptoms of pruritus and weakness. The patient was therefore admitted to The First Central Hospital of Tianjin. Upon admission, a physical examination revealed rashes on the extremities, but no sternal pain or tenderness, and no peripheral lymphadenopathy or hepatosplenomegaly. The 

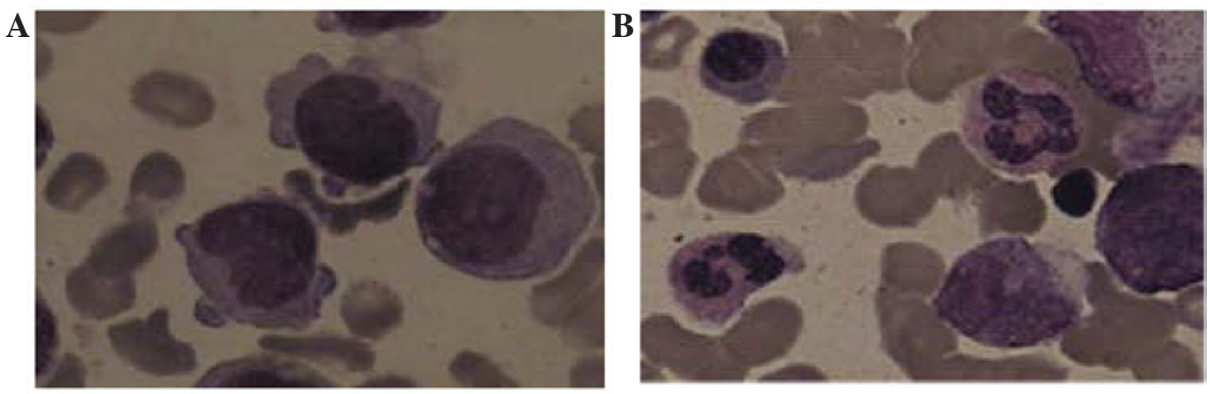

Figure 1. Digital images revealing Wright and Giemsa-stained bone marrow monoblasts and promonocytes (A) prior to and (B) following treatment with the combination regimen (magnification, $\mathrm{x} 1,000$ ).
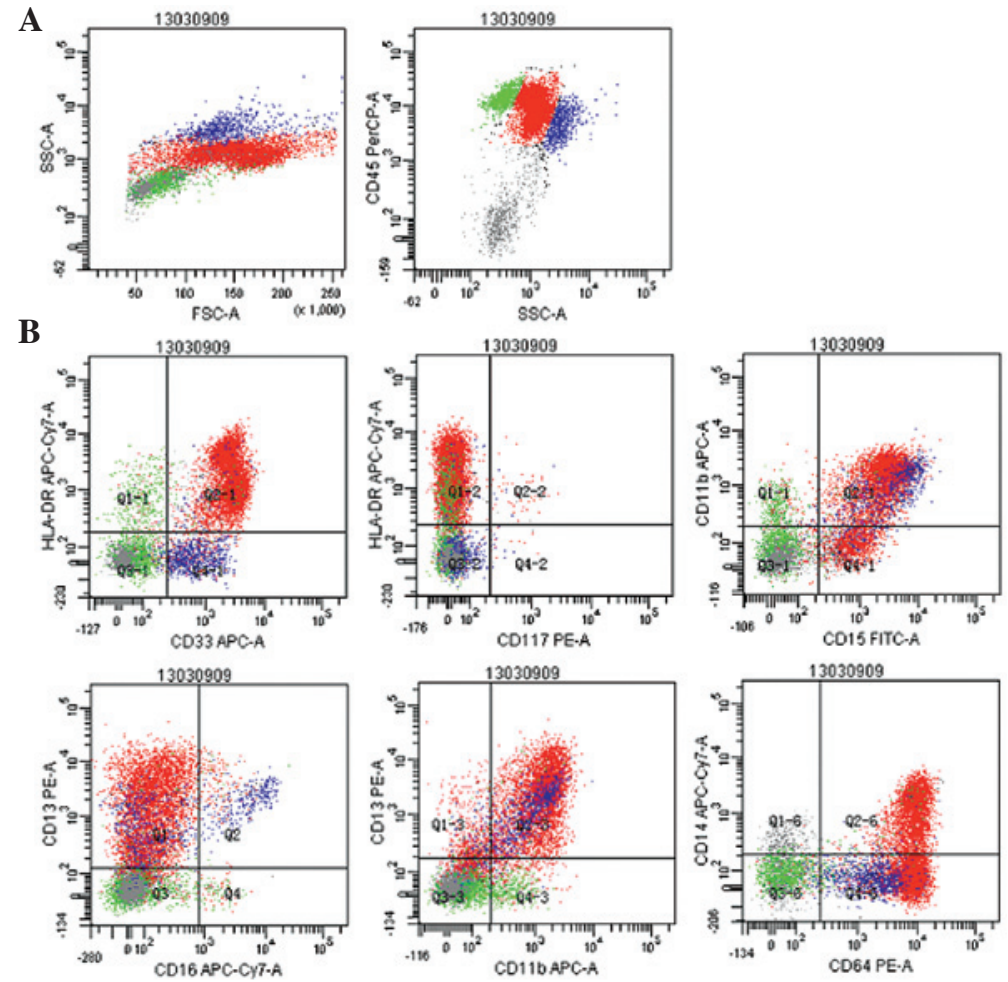

Figure 2. Flow cytometry scatter plots revealing the gating strategy with (A) cluster of differentiation (CD)45/side-scattered light and (B) the ratio of monoblasts (72.3\%), and their expression of the myelomonocytic antigens, human leukocyte antigen-DR, CD15, CD4, CD13, CD33, CD38, CD64, D11b, CD14 and CD56.

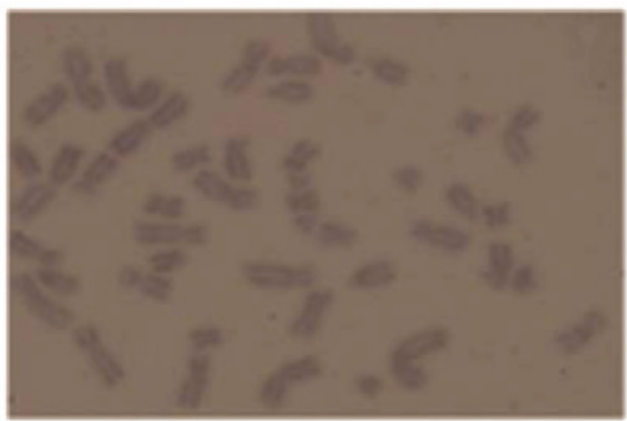

Figure 3. Cytogenetic analysis revealing Y chromosomal loss, which was the single anomaly identified in the patient $(45, \mathrm{X},-\mathrm{Y}[4] / 46, \mathrm{XY}[16])$ (magnification, $\mathrm{x} 1,000)$

routine blood test identified a $\mathrm{Hb}$ level of $106 \mathrm{~g} / \mathrm{l}$, a WBC count of $39.52 \times 10^{9} / 1$ and a platelet count of $81 \times 10^{9} / 1$. A diagnosis of
AML was suspected. A bone marrow aspirate examination revealed a monoblast percentage of $38 \%$ and a promonocyte percentage of $55 \%$ (Fig. 1A), values which were significantly higher than the threshold of blasts $(>20 \%)$ required in the bone marrow for a diagnosis of AML, according to the 2008 World Health Organization (WHO) (6) AML classification systems. Immunohistochemical analysis revealed strong staining signals for myeloperoxidase in the cytoplasm of the blasts, and the myeloid lineage markers, human leukocyte antigen-DR and cluster of differentiation (CD)15, CD4, CD13, CD33, CD38, CD64, CD11b, CD14 and CD56, were detected on the surface of the monoblasts by multiparameter flow cytometry (Fig. 2). There was no evidence of a JAK2V617F mutation or a $\mathrm{BCR} / \mathrm{ABL}$ fusion transcript. In addition to the bone marrow aspiration assessment, immunohistochemical staining and flow cytometric analyses, single nucleotide polymorphism (SNP) arrays were performed. Cytogenetic abnormalities, including Y chromosome loss (Fig. 3) and uniparental disomy (UPD) on 


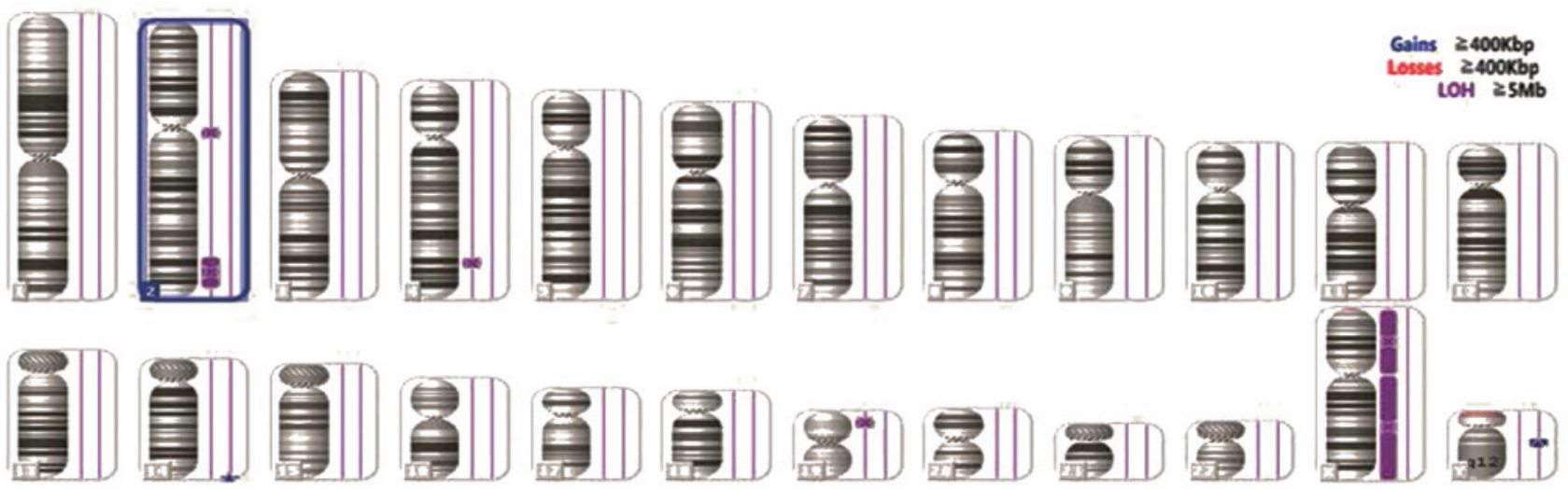

Figure 4. Uniparental disomy of chromosomes 4q, 2q and 19p, and a copy number variation of 14q. Yq chromosome deletion detected by single nucleotide polymorphism analysis. $\mathrm{LOH}$, loss of heterozygosity.

chromosomes 4q, 2q and 19p (Fig. 4) were detected. Considering the two-year history of monocytosis, the patient was diagnosed with AML transformed from CMML together with a rare genetic abnormality (6).

The patient was treated with $1,000 \mathrm{mg}$ imipenem every $8 \mathrm{~h}$ for eight days, and $500 \mathrm{mg}$ hydroxycarbamide every $12 \mathrm{~h}$ for four days. Subsequently, a combination treatment regimen consisting of decitabine and CAG was initiated. In total, $15 \mathrm{mg} / \mathrm{m}^{2}$ decitabine was administered daily on days one to five, $20 \mathrm{mg}$ aclarubicin daily on days three to six, $20 \mathrm{mg} / \mathrm{m}^{2}$ cytarabine twice daily on days three to 16 and $150 \mu \mathrm{g}$ G-CSF daily from day two until the attainment of a normal WBC count. At the end of the low-dose regimen, the patient achieved a CR and experienced less severe adverse events, specifically severe infections. The values of the major test parameters were normal or close to normal, and included a $\mathrm{Hb}$ level of $117 \mathrm{~g} / \mathrm{l}$, a WBC count of $4.52 \times 10^{9} / 1$, a platelet count of $370 \times 10^{9} / 1$, a monocyte percentage of $6.5 \%$ and a monoblast percentage of $1.5 \%$ (Fig. 1B). Administration of the regimen was continued. Subsequent to four cycles of treatment with decitabine in combination with CAG, the patient achieved a successful clinical remission and demonstrated no evidence of AML relapse for nine months. Written informed consent was obtained from the patient's family for the publication of the case report and the accompanying images.

\section{Discussion}

CMML, defined by the 2008 WHO classification of myeloid neoplasms as a clonal hematopoietic stem cell disorder (6), is currently classified under a new division of myeloid neoplasms, the MDS/myeloproliferative neoplasm disorders. These disorders exhibit dysplastic and proliferative features. Cytogenetics is considered to be one of the most valuable determinants used during the risk classification and prognostication of AML $(7,8)$. Previous studies have revealed that SNP arrays can identify chromosomal markers that cannot be detected by conventional cytogenetics $(9,10)$. In general, specific cytogenetic alterations cannot be identified in patients with CMML by chromosome banding analysis alone. The most common single chromosomal abnormalities in cases of CMML are monosomy 7 (3.9-8.5\%), trisomy $8(4.1-7.8 \%)$ and other abnormalities, which may include complex karyotypes (4.4-6.3\%), isochromosome 17 (1-2\%), trisomy 21 (1-2\%) and deletion of $5 \mathrm{q}(1.5 \%)$ (11-13). Abnormal karyotypes have been reported in 20-40\% of CMML cases, and include abnormalities shared with other myeloid neoplasms (14). According to a previous study involving 140 patients (10), acquired somatic UPD is not uncommon in primary (29\%) and secondary (35\%) AML. Although AML with UPD q4 is rarely reported (15), another previous study (16) revealed an association between UPD $4 \mathrm{q}$ and CMML. Based on the aforementioned criteria and studies, the patient in the present study was diagnosed with AML transformed from CMML.

CMML remains to be a challenging malignancy to treat, and for this reason, the median survival rate for patients ranges between 12 and 18 months. If CMML progresses to AML, patients exhibit poor prognoses, face limited treatment options and on average, demonstrate survival rates of only a few months (17). The only potential curative treatment for patients with CMML is allogeneic HSCT, however, this particular approach is usually unsuitable for elderly individuals. Treatment with the DNA methyltransferase inhibitors, azacitidine and decitabine, has been extensively studied for the management of AML and MDS (18-21). Two mechanisms are believed to underlie the action of decitabine. First, decitabine incorporates into the DNA following phosphorylation, without the need for reduction. Secondly, decitabine does not incorporate into RNA, and inhibits DNA methyltransferase 2 (22). A Japanese study, which included AML patients $\geq 60$ years old, revealed that when a 10-day decitabine regimen was administered and then repeated following an interval of 4-5 days, CR and median survival rates of $47 \%$ and 12.7 months, respectively, were achieved subsequent to an average of three cycles of therapy (23). The CAG regimen, which combines G-CSF with low doses of cytarabine and aclarubicin, was first used by a Japanese study in 1995, and was reported to be a chemotherapy option for AML (24). In this regimen, aclarubicin is effective regardless of multi-drug resistance gene status, and G-CSF acts to enhance the transition of resting $\mathrm{G}_{0}$ phase AML cells into the cell cycle. The CAG regimen has been used for relapsed or refractory AML and MDS patients, and also specifically for elderly patients. The high response rates and good tolerability of this regimen is observed not only in 
relapsed or refractory cases, but also in previously untreated patients $(25,26)$. Therefore, for these reasons, the combination of decitabine and CAG was selected to treat the patient in the present study.

Due to the conjecture diagnosis of CMML in the present study, the regimen of decitabine in combination with CAG was selected. This regimen consisted of low-dose decitabine $\left(15 \mathrm{mg} / \mathrm{m}^{2}\right)$, aclarubicin $\left(15 \mathrm{mg} / \mathrm{m}^{2}\right.$ for four days) and low-dose cytarabine $\left(15 \mathrm{mg} / \mathrm{m}^{2}\right.$, every $12 \mathrm{~h}$ for 10 days). The patient achieved a CR following only one such low-dose regimen, and experienced less severe adverse events, specifically severe infections. Subsequent to a total of four cycles of treatment with decitabine in combination with CAG, the patient achieved a successful clinical remission. In conclusion, a combination of low-dose decitabine and CAG may offer a novel and potentially effective treatment regimen for cases of $\mathrm{AML}$ transformed from CMML.

\section{Acknowledgments}

The present study was supported by grants from the Tianjin Municipal Health Bureau of Science and Technology Fund 2013 Project (no. 13KG106).

\section{References}

1. Döhner H, Estey EH, Amadori S, et al; European LeukemiaNet: Diagnosis and management of acute myeloid leukemia in adults: recommendations from an international expert panel, on behalf of the European LeukemiaNet. Blood 115: 453-474, 2010.

2. Dombret H, Raffoux E and Gardin C: New insights in the management of elderly patients with acute myeloid leukemia. Curr Opin Oncol 21: 589-593, 2009.

3. Lancet JE, Willman CL and Bennett JM: Acute myelogenous leukemia and aging. Clinical interactions. Hematol Oncol Clin North Am 14: 251-267, 2000.

4. Lin TL and Levy MY: Acute myeloid leukemia: focus on novel therapeutic strategies. Clin Med Insights Oncol 6: 205-217, 2012

5. Ungewickell A and Medeiros BC: Novel agents in acute myeloid leukemia. Int J Hematol 96: 178-185, 2012.

6. Vardiman JW, Thiele J, Arber DA, et al: The 2008 revision of the World Health Organization (WHO) classification of myeloid neoplasms and acute leukemia: rationale and important changes. Blood 114: 937-951, 2009.

7. Grimwade D, Walker H, Oliver F, et al: The importance of diagnostic cytogenetics on outcome in AML: analysis of 1,612 patients entered into the MRC AML 10 trial. The Medical Research Council Adult and Children's Leukaemia Working Parties. Blood 92: 2322-2333, 1998.

8. Löwenberg B: Diagnosis and prognosis in acute myeloid leukemia - the art of distinction. N Engl J Med 358: 1960-1962, 2008.

9. Mohamedali A, Gäken J, Twine NA, et al: Prevalence and prognostic significance of allelic imbalance by single-nucleotide polymorphism analysis in low-risk myelodysplastic syndromes. Blood 110: 3365-3373, 2007.

10. Tiu RV, Gondek LP, O'Keefe CL, et al: New lesions detected by single nucleotide polymorphism array-based chromosomal analysis have important clinical impact in acute myeloid leukemia. J Clin Oncol 27: 5219-5226, 2009.
11. Bacher U, Haferlach T, Kern W, et al: Conventional cytogenetics of myeloproliferative diseases other than CML contribute valid information. Ann Hematol 84: 250-257, 2005.

12. Haase D, Germing U, Schanz J, et al: New insights into the prognostic impact of the karyotype in MDS and correlation with subtypes: Evidence from a core dataset of 2124 patients. Blood 110: 4385-4395, 2007.

13. Kohlmann A, Grossmann V, Klein HU, et al: Next-generation sequencing technology reveals a characteristic pattern of molecular mutations in $72.8 \%$ of chronic myelomonocytic leukemia by detecting frequent alterations in TET2, CBL, RAS, and RUNX1. J Clin Oncol 28: 3858-3865, 2010.

14. Orazi A and Germing U: The myelodysplastic/myeloproliferative neoplasms: myeloproliferative diseases with dysplastic features. Leukemia 22: 1308-1319, 2008.

15. Raghavan M, Smith LL, Lillington DM, et al: Segmental uniparental disomy is a commonly acquired genetic event in relapsed acute myeloid leukemia. Blood 112: 814-821, 2008.

16. Gondek LP, Tiu R, O'Keefe CL, et al: Chromosomal lesions and uniparental disomy detected by SNP arrays in MDS, MDS/MPD, and MDS-derived AML. Blood 111: 1534-1542, 2008.

17. Parikh SA and Tefferi A: Chronic myelomonocytic leukemia: 2012 update on diagnosis, risk stratification, and management. Am J Hematol 87: 610-619, 2012.

18. Kantarjian H, Oki Y, Garcia-Manero G, et al: Results of a randomized study of 3 schedules of low-dose decitabine in higher-risk myelodysplastic syndrome and chronic myelomonocytic leukemia. Blood 109: 52-57, 2007.

19. Steensma DP, Baer MR, Slack JL, et al: Multicenter study of decitabine administered daily for 5 days every 4 weeks to adults with myelodysplastic syndromes: the alternative dosing for outpatient treatment (ADOPT) trial. J Clin Oncol 27: 3842-3848, 2009.

20. de Lima M, Giralt S, Thall PF, et al: Maintenance therapy with low-dose azacitidine after allogeneic hematopoietic stem cell transplantation for recurrent acute myelogenous leukemia or myelodysplastic syndrome: a dose and schedule finding study. Cancer 116: 5420-5431, 2010.

21. Baer MR and Gojo I: Novel agents for the treatment of acute myeloid leukemia in the older patient. J Natl Compr Canc Netw 9: 331-335, 2011.

22. Oki Y, Kondo Y, Yamamoto K, et al: Phase I/II study of decitabine in patients with myelodysplastic syndrome: a multi-center study in Japan. Cancer Sci 103: 1839-1847, 2012.

23. Blum W, Garzon R, Klisovic RB, et al: Clinical response and miR-29b predictive significance in older AML patients treated with a 10-day schedule of decitabine. Proc Natl Acad Sci USA 107: 7473-7478, 2010.

24. Yamada K, Furusawa S, Saito K, et al: Concurrent use of granulocyte colony-stimulating factor with low-dose cytosine arabinoside and aclarubicin for previously treated acute myelogenous leukemia: a pilot study. Leukemia 9: 10-14, 1995.

25. Saito K, Nakamura Y, Aoyagi M, et al: Low-dose cytarabine and aclarubicin in combination with granulocyte colony-stimulating factor (CAG regimen) for previously treated patients with relapsed or primary resistant acute myelogenous leukemia (AML) and previously untreated elderly patients with AML, secondary AML, and refractory anemia with excess blasts in transformation. Int J Hematol 71: 238-244, 2000.

26. Suzushima H, Wada N, Yamasaki H, et al: Low-dose cytarabine and aclarubicin in combination with granulocyte colony-stimulating factor for elderly patients with previously untreated acute myeloid leukemia. Leuk Res 34: 610-614, 2010. 